\title{
STRONTIUM-90 IN HUMAN DIET
}

$\mathbf{R}$ ESPONSIBILITY for estimating the contamination of food by radioactive fall-out has now been transferred to the Agricultural Research Council. The report, "Strontium-90 in Human Diet in the United Kingdom, 1958" (London: H.M. Stationery Office, 1959. 4s.), thus continues the series formerly issued by the Atomic Energy Authority.

The general level of radioactivity in food in the United Kingdom for 1958 was small, comparable with that found in the United States, and well below any danger-level. Most of the radioactivity in milk and dairy products, bread and flour, and in leafy and root vegetables, was brought about by the deposition of radioactive debris on the leaves of herbage and crop plants during periods of rain, followed by foliar absorption of (in particular) strontium-90. Only the material deposited during the two months before herbage was eaten by cows or before crops were harvested appeared afterwards in appreciable amounts in human food. Once the debris penetrated into the soil, its 'availability' to plants was greatly reduced. Consequently, the accumulation of available strontium-90 in the soil since nuclear weapon testing commenced in 1945 had been slight.

The radioactivity of milk was highest in western areas of Britain and appeared to be related to the distribution of rainfall. The level of radioactivity increased in all areas during the latter half of 1958 , partly as a result of an unusually high rainfall and partly of an increase in the number of nuclear tests.

Examination of certain upland areas that are characterized by high rainfall and by slow growth of herbage had shown that milk from these areas often contained very high levels of strontium-90. This could not be accounted for entirely on the basis of high rainfall, low soil-calcium, and low production of herbage per acre. It was thought that strontium-90 must become entrapped in the mat of vegetation and roots at the base of the sward, and be held available to the plant in successive seasons, in a way not observed on lowland pastures.

The report shows that when strontium was absorbed from the diet, it tended to replace calcium in bone tissue. High levels of strontium-90 in bone could damage the bone or bone marrow, ultimately causing tumours, leukæmia, or other bone diseases.
However, it appeared that the replacement of calcium by strontium-90 in new bone tissue was governed not by the amount of strontium-90 in the diet but by its proportion relative to calcium (expressed as micromicrocuries of strontium-90 per gm. calcium) Furthermore, the human body, in absorbing mineral substances from the digestive tract, discriminated against strontium, so that the ratio of strontium-90 to calcium which was found in bone was only onequarter of that in the food eaten.

The report also discusses other factors which tended to reduce the ratio of strontium to calcium in the diet. Thus, the cow discriminated against strontium when producing milk from grass, so that the proportion of strontium-90 to calcium in milk was only one-seventh of that in the herbage eaten. Leafy vegetables appeared to absorb strontium less efficiently than herbage, and contained correspondingly less radioactive material. Although wheat contained a fairly high proportion of strontium.90, milling, as in the preparation of white flour, removed much of the calcium and strontium in bran and offal, while the subsequent addition of strontium-free chalk as a calcium supplement further reduced the proportion of strontium to calcium in bread and flour products. The report noted that the ratio of strontium-90 to calcium in diets based on wholemeal bread was likely to be higher than average, as there was no legal requirement to add chalk to wholemeal flours, while the milling process did not tend to remove any of the strontium contained in the grain.

However, even on the most unusual food and living in the wettest area, no one in the United Kingdom was likely to consume a diet containing more than $23 \mu \mu c$. of strontium-90 per gm. calcium, about half the ratio $(40 \mu \mu \mathrm{c}$.$) at which a Medical Research$ Council Committee thought that 'immediate consideration' should be given to the problem. It is emphasized that no evidence had been found of such a diet being consumed by anyone. The amount of strontium-90 per gm. calcium in the average diet was about $6 \mu \mu \mathrm{c}$, and, provided that the rate of deposition of the radioactive dust did not greatly increase in the future, either as a result of meteorological factors or because of further testing of nuclear devices, such a level should give no eause for anxiety.

J. M. A. Tillex

\section{IMMEDIATE AND LOW-LEVEL EFFECTS OF IONIZING RADIATIONS}

\begin{abstract}
$\mathrm{T}$ HE biological effects of low doses of ionizing radiations, a topic of obvious interest and impor. tance, formed one of the main themes of a symposium held in Venice, June 22-26, under the joint sponsorship of Unesco, the International Atomic Energy Agency and the Comitato Nazionale per le Ricerche Nucleari of Italy. The Organizing Committee included Prof. Z. M. Bacq (Belgium); Profs. E. Boeri and A. A. Buzzati-Traverso (Italy); and Dr. A. Hollaender (United States). Those invited were fortunate in being able to take part in a conference of which content and programme arrangements were of a high
\end{abstract}

order, and which was held in the beautiful surroundings of the Fondazione Giorgio Cini, on the Isola di San Giorgio Maggiore. Each of the nine sessions was arranged to contain only a few papers, so that there was ample time for discussion and for a few short communications which were relevant to the main themes. Sixteen countries, and a wide range of scientific disciplines, were represented among the 116 research workers who took part.

The symposium opened with a review of certain aspects of quantitative radiobiology by K. G. Zimmer (Kernreaktor, Karlsruhe, Germany). After diseussing 
critically some of the postulated mechanisms of the biological action of ionizing radiations, he went on to describe recent results obtained by the use of microwave spectroscopy. This topic, discussed also by J.S. Kirby-Smith (Oak Ridge, United States), is of great interest because persistent magnetic centres can be observed in irradiated biological materials of low water content, and the signals are modified by environmental factors which are known also to modify the biological effects of radiation. However, caution is necessary in interpreting the results obtained by instruments currently in use, since the signals observed arise from about $10^{10}$ times as many ionizing events as those which in many cases initiate biological damage.

New techniques which have recently been developed for studying cells in mitosis have enabled investigators to undertake the difficult task of observing quantitatively the induction of chromosomal abnormalities in human cells. M. A. Bender (Oak Ridge, United States) had examined effects on human cells in tissue culture, and also on monkey cells in vivo, using bone marrow. The cells irradiated in vivo gave a somewhat lower yield of ehromosome aberrations. Doses down to $25 \mathrm{r}$. were used in these studies.

The effects of considerably lower doses on human cells were observable by M. Ingram (University of Rochester, United States), who had found significant increases in the number of binucleate lymphocytes present in the blood of persons exposed to doses considered to be in the 'tolerance' range. Although ionizing radiation is not the only agent which can bring this about, it was of interest that in a field investigation of uranium miners, a higher count of binucleate lymphocytes was found in the blood of the control group of coal miners, who had been subjected to regular routine diagnostic X-ray examination! Another paper on effects of X-irradiation on the blood picture of mammals was given by S. Hajdukovic (Institute for Nuclear Sciences, Yugoslavia), who used as his test effect the increase in the number of reticulocytes released into peripheral blood. $\mathrm{He}$ reported that increases were also obtained when serum from irradiated animals was injected into nonirradiated ones, the effect not being species-specific. These changes were observed fairly early after the irradiation.

The subject of chemical protection against biological effects of ionizing radiation was reviewed by D. W. van Bekkum (National Defence Research Council, Holland), who discussed different groups of protective substances and critically examined possible mechanisms of action. R. Brinkman (State University, Groningen, Holland) described techniques for examining the protective effect of chemicals against radiation-induced changes which could be measured very soon after low doses of irradiation. These included measurements of the viscosity of synovial fluid, and of intradermal pressure. Effects of irradiation could be observed within one second, and serotonin injected intradermally was the most effective of the protective substances used. M. Ebert and A. Howard (Medical Research Council and British Empire Cancer Campaign, Great Britain) described some of their latest findings with inert gases. These gases suppressed the enhancing action of oxygen when used at pressures above atmospheric, but were less effective in the cold than at room temperature. J. F. H. Maisin (University of Louvain, Belgium) had found that small doses of radiation could themselves protect against the damaging effect of larger doses. Yeast cells were exposed for long periods to continuous irradiation which was not itself sufficient to kill the cells ; thereafter, larger doses were required to produce a given killing effect on these than on control cells. Rats which had been exposed to radiation in utero or as new-borns were more resistant than controls to radiation given afterwards.

Various types of immediate response to irradiation had been observed by $\mathrm{O}$. Hug (International Atomic Energy Agency, Austria), who showed a film which demonstrated reflex reactions of snails, sea urchins and ants. It was clear that an immediate effect of radiation could be observed with nerve tissue, long thought to be comparatively insensitive to its action. A different type of immediate response was reported by A. Forssberg (Institute of Radiophysics, Sweden), who has observed reversible effects of doses as low as $10^{-3} \mathrm{r}$. on the fungus Phycomyces blakesleeanus, the growth-rate of the sporangiophore being immediately reduced. Depression of growth-rate was accompanied by an increase in the level of acid-labile phosphorus, and a slightly delayed increase in lactic acid. It was suggested that the use of adenosine triphosphate might be blocked by the radiation.

Radiobiologists continue to search for the biochemical links between the absorption of ionizing energy and the manifestation of the effects observed, and various approaches were reported. P. Alexander (Chester Beatty Research Institute, Great Britain) gave an account of physico-chemical studies on effects of radiation on deoxyribonucleic acid in vitro and in herring sperm, including a discussion of the phenomenon of cross-linking. K. I. Altman (University of Rochester, United States) had studied the breakdown of muscle collagen in rats previously fed with labelled amino-acids. Whole-body irradiation with lethal doses was followed by a reduction in the hydroxylation of proline and an increase in that of its precursor.

A. Chevallier and S. Manuel (University of Strasbourg, Franee) found that one result of radiation which could be measured within a short time was a drop in the ascorbic-acid content of almost all tissues, particularly the spleen. While this was true of animals irradiated as a whole, spleen slices irradiated in vitro did not demonstrate the phenomenon, nor was it observed when only the exteriorized spleen was irradiated. The authors concluded that the drop in ascorbic acid in the spleen depended on effects on other organs. P. Mandel and P. Chambon (University of Strasbourg, France) reported studies on ribonucleie acid synthesis in rat spleen after whole-body irradiation. Accumulated nucleotides were found in this organ from 12 hours after irradiation. R. Goutier, M. Goutier-Pirotte and P. Ciccarone (University of Liège, Belgium) examined an effect which occurred soon after the comparatively low dose of $150 \mathrm{r}$. (whole-body) to rats. Changes in the deoxyribonuclease activity of the spleen could be detected after half an hour. The activity of the extracted enzyme depended critically upon the methods used in preparing the sample. The authors considered that the effect was due to a change in the enzyme molecule, and not to the effect of the irradiation on enzyme distribution.

H. I. Adler (Oak Ridge, United States) reported on observations with a variant of Escherichia coli which did not synthesize catalase. One effect of irradiation was to sensitize the cells to the action of hydrogen peroxide. Irradiated cells exposed to its action were killed, although they would otherwise have survived. 
Bacteria were used in two studies of the effects of low doses of radiation. Marcovich (Institut du Radium, France) had examined the induction of lysogenic bacteria, and concluded that the passage of a single ionizing particle through a cell was sufficient to bring about this effect. M. Demerec (Carnegie Institute of Washington, United States) made use of three biochemical mutations which occurred spontaneously with very low frequency, so that the genetic effects of doses as low as $8.5 \mathrm{r}$. could be assessed. In all three cases, the number of mutations induced was proportional to the dose at low doses, though the doses required to produce a given frequency of mutations differed for the three mutations chosen for study.

New observations on the genetic effects of ionizing radiation on mice were reported by W. L. Russell (Oak Ridge, United States). These have confirmed his previous report that if a dose in the range 200 $600 \mathrm{r}$. was delivered at $80 \mathrm{r}$./min. more mutations were induced in spermatogonia than if the same dose was delivered continuously as 'ehronic' irradiation at $90 \mathrm{r}$. /week or less. This did not apply to mutations induced in spermatozoa. The genetic effect of a single dose on oocytes was greater than on spermatogonia, whereas the reverse was true of chronic irradiation. L. B. Russell (Oak Ridge, United States) reported that chronic irradiation was less effective than an acute dose, delivered to embryos in their most sensitive stage, in bringing about still-births and neonatal deaths.

Various tests of radiation damage were used by L. J. Cole (Naval Radiobiological Defense Labora. tory, United States) in comparing effects of single exposures and 'chronic' or fractionated radiation. Single doses were less effective in inducing leukæmia or shortening the life span; but the effects of chronic irradiation doses on the fertility of female mice were much lower than were single doses. The effect of as little as $25 \mathrm{r}$. in a single dose could be detected in weanlings. The injection of bone marrow, which protected against the lethal effects of $800 \mathrm{r}$., did not protect against loss of fertility.

Immunological aspects of tissue transplantation after $300 \mathrm{r}$. of X-rays had been studied by $\mathrm{P}$. C. Koller and S. Doak (Chester Beatty Research Institute, Great Britain). After fifty days, the immune response of some 'chimaeras' had reverted to the host type, others had retained the immunity of the donor, and yet others gave a mixed response.

This very brief account of the subjects discussed at the symposium should make it evident how wide a range of materials, and how many different approaches, are being used in attempts to clarify some of the outstanding problems in radiation biology. The proceedings of the symposium are to be published as a supplement to the International Journal of Radiation Biology.
TikVah AlPer

\section{NUCLEAR FORCES AND THE FEW-NUCLEON PROBLEM}

$\mathrm{M}$ ORE than 250 nuclear physicists, including about 100 delegates from fifteen countries overseas, attended the international conference, which was held at the Physics Department, University College, London, during July 8-11. A conference on the behaviour of light nuclei had not taken place for several years and was initiated by physicists at Los Alamos and University College. As was stressed by Prof. H. S. W. Massey (University College), who opened the conference, although the original intention was to emphasize the few-nucleon problem, discussion of nuclear forces had inevitably to be included.

The conference consisted of five main sessions, the first and longest being entirely devoted to the primary two-nucleon interaction. In this session review papers were given by Profs. R. E. Marshak (Rochester), R. Wilson (Harvard), K. A. Brueckner (Pennsylvania) and G. F. Chew (Berkeley) on both the experimental and theoretical status of the problem. Interest centred on many sets of measurements, including triple scattering and correlation experiments, as well as more accurate cross-section measurements at various energies and on their interpretation in terms of the $S$-matrix. Prof. G. Breit (Yale) presented an extensive search for phase-shift fits to the scattering data up to $340 \mathrm{MeV}$., while comparison was made with phase-shifts derived from various phenomenological potentials by Prof. Marshak. There was discussion on both the necessity and theoretical justification for including spin-orbit and other velocity-dependent potentials in the two-body force. The experimental papers were concerned with recent triple scattering measurements in $p-p$ scattering at Rochester and Harwell and with $p-p$ angular distributions at Minnesota. Also $n-p$ angular dis- tributions and polarization measurements from 20-120 MeV. were reported by Dr. J. J. Thresher (Harwell).

Dr. J. Iwadare (Kyoto) summarized the recent work done in Japan on the meson-theoretical twonucleon interaction and its comparison with experimental data. This was followed by Prof. Chew's paper, which reviewed recent work on the meson field-theoretical approach to the two-body problem starting from the Mandelstam conjecture on the analytical form of the scattering amplitude. The inclusion of the pion-pion interaction within the context of dispersion relations seems to be the next step in the long struggle to obtain meaningful results from the meson theory of nuclear forces proposed by Yukawa in 1935 .

Prof. Yukawa was chairman for the beginning of the second session, on the scattering of nucleons by light nuclei at high energy. A review paper on the impulse approximation by Dr. H. McManus (Chalk River) was followed by applications of this approach to the $n-d$ and $p-d$ case by Drs. L. Castillejo (Birmingham), R. Phillips (Harwell) and by Japanese workers. The problem was examined from the point of view of dispersion theory in a paper by Goldberger, Halpern and Blankenbecler (Princeton), and corrections due to multiple scattering were considered by Prof. R. J. Glanber (Harvard). In this session there were reports by Drs. A. M. Cormack, T. C. Griffith and G. Huxtable on experiments done at Harvard, University College and Harwell respectively on $p-d$ and $p-\alpha$ scattering at energies between 50 and $150 \mathrm{MeV}$.

The session on photonuclear reactions with light nuclei opened with a review by Dr. D. Dixon 